\title{
Visual space-time interactions: Effects of adapting to spatial frequencies on temporal sensitivity
}

\author{
MARISA CARRASCO \\ Wesleyan University, Middletown, Connecticut
}

\begin{abstract}
To study how adaptation to spatial frequency patterns affects temporal sensitivity in vision, observers were selectively adapted for $4 \mathrm{~min}$ to either a high-or a low-spatial-frequency sinusoidal grating (12 and $2 \mathrm{cpd}$, respectively). Their sensitivities to modulation of a blurred patch at high or low temporal frequencies $(12 \mathrm{~Hz}$ and $2 \mathrm{~Hz}$, respectively) were measured, before and after the adaptation period, by using the yes/no task of signal detection theory. The data consistently indicated that spatial adaptation differentially affected the observers' sensitivities to temporal signals. Specifically, when the observers were adapted to low spatial frequencies, their sensitivity to low temporal frequencies was reduced; when they were adapted to high spatial frequencies, their sensitivity to high temporal frequencies was increased. These results have implications for the psychophysical measurements of temporal and spatial sensitivity, as well as for the issue of the separability of spatial and temporal properties of individual channels.
\end{abstract}

With respect to human spatial vision, it is now generally accepted that information undergoes parallel processing by a number of different channels, each tuned for orientation and spatial frequency (see, e.g., R. L. DeValois \& K. K. DeValois, 1988; Graham, 1985; Olzak $\&$ Thomas, 1986). The human visual system seems also to be tuned for the detection of specific temporal frequencies or bands of frequencies (see, e.g., M. Green, 1981; Hess \& Plant, 1985; Kelly, 1961). It has been found that over a wide range of average intensities the temporal contrast sensitivity functions (TCSFs) are generally similar in shape to the spatial contrast sensitivity functions; both drop off slowly toward low frequencies and quickly toward high frequencies. Although for the most part the two sensitivities have been studied separately, it has been recently suggested that a major task for future vision research will be the integration of models of spatial sensitivity and temporal sensitivity (Olzak \& Thomas, 1986; Watson, 1986). The experiment reported here was designed to contribute to this integration.

Several authors have explored the effects of spatial factors on TCSFs. More concretely, four distinct aspects of the spatial configuration of the stimuli have been

I thank Ron Kinchla, my thesis advisor, for guidance and support throughout this project. I am also indebted to Bill Prinzmetal, Carl Sherrick, and Charlie Gross, members of my dissertation committee. Jim Fernandez, Cathi Best, C. W. Tyler, and two anonymous reviewers made helpful comments on drafts of the article; the computer program was written by Derek Gross. This experiment was part of the dissertation submitted by the author in partial fulfillment of the requirements for the $\mathrm{PhD}$ degree at Princeton University. Some of the work was presented at the 12th European Conference on Visual Perception (September 1989). Correspondence should be addressed to Marisa Carrasco, Department of Psychology, Wesleyan University, Middletown, CT 06457. shown to reduce sensitivity at low temporal frequencies: enlarging the size of a target, removing its surround, blurring its edges, or lowering its spatial frequencies (see Watson, 1986).

The spatiotemporal characteristics of the visual system have also been studied by considering the relation of temporal and spatial sensitivities in terms of their separability (see, e.g., Kelly, 1966; Koenderink \& van Doorn, 1979; Robson, 1966). Sensitivity is separable along two dimensions if it is given by the product of their sensitivities along the individual dimensions (see, e.g., Watson, 1986). Spatiotemporal separability implies that spatial tuning is altered at most by a scalar multiple with temporal frequency and vice versa; that is, the shape of the spatial tuning curve remains constant regardless of temporal frequency (Fleet, Hallett, \& Jepson, 1985; Watson, 1986; Wilson, 1980). If this condition is met, the responses of the underlying mechanisms to spatial and temporal modulation are said to be independent.

As Lehky (1985) has clearly expressed, "whether or not separability occurs is an important question, for without it any experimental determination of spatial(temporal-) tuning curves would hold true only for the particular temporal (spatial) conditions of that particular experiment, whereas with separability measurements have a more general validity" (p. 1260). An essential task would then be to study specifically how the domains interact, and measurements of the spatial or temporal sensitivities would have to take the other domain into consideration.

It is generally accepted that the spatiotemporal surface as a whole is not separable. At high temporal frequencies, the effect of modulation on contrast sensitivity is independent of spatial frequency, whereas at low temporal 
frequencies, sensitivity to low spatial frequencies is selectively reduced; that is, at low frequencies the two functions are inseparable (see, e.g., Koenderink \& van Doorn, 1979; Olzak \& Thomas, 1986; Robson, 1966; Watson, 1986). However, there is some controversy regarding the possibility of describing the system as a collection of channels whose spatial and temporal properties are separable.

Some authors maintain that the response of individual human spatiotemporal mechanisms is separable into a product of spatial and temporal functions (e.g., Harris, 1984; Lehky, 1985; Wilson, 1980). Studies of both simple and complex striate neurons in the cat provide neurophysiological evidence for this position (Tolhurst \& Movshon, 1975). Other authors, however, have questioned the separability of these visual mechanisms (e.g., Fleet et al., 1985). Retinal ganglion cells in the cat show significant changes in spatial tuning when measured under different temporal conditions (Enroth-Cugell \& Lennie, 1975). Furthermore, inseparable behavior has been verified for ganglion $x$-cells and y-cells in cats (Derrington \& Lennie, 1982), and in both the phase and the amplitude components of $x$-cells' responses (Dawis, Shapley, Kaplan, \& Tranchina, 1984). In each case, either the surround mechanism of the receptive field has a slightly longer delay than the center mechanism, or the signals from the surround undergo somewhat more temporal filtering than those from the center (Fleet et al., 1985).

A conventional way of looking at the separability issue in individual channels is to use the psychophysical procedure of selective adaptation. By demonstrating that prolonged exposure to one type of stimulus reduces sensitivity only to certain other stimuli, this procedure has shown selectivity for spatial frequency, orientation (see, e.g., Blakemore \& Campbell, 1969), and temporal frequency (see, e.g., Smith, 1970). In three experiments, selective adaptation effects between the spatial and temporal domains have been found. Two of these show some effects of adapting to temporal frequencies on the perception of spatial frequencies (M. Green, 1981; Parker, 1981). The third shows some effects of adapting to spatial frequencies on the detection of motion (Pantle, 1970).

The present study was carried out to explore whether adaptation to spatial frequency patterns affects observers' sensitivities to temporal changes in luminance (flicker). Observers' sensitivities to low- or high-frequency temporal stimuli were measured before and after adaptation to spatial gratings of low or high frequency.

\section{GENERAL METHOD}

To assess temporal sensitivity, a detection task based on signal detection theory (SDT) was used, thus allowing the measurement of very small shifts in sensitivity to temporal patterns independently of judgmental standards (decision criteria). There were four phases: In Phase 1, the TCSFs of 4 observers were estimated. In Phase 2, stimulus amplitudes were adjusted for each observer to yield similar discriminability values. In Phase 3 , the effects of spatial adaptation on temporal sensitivity were explored by using a detection task based on SDT. And in Phase 4, a receiver operating characteristic (ROC) was generated by manipulating the instructions given to observers in order to determine whether there were additive effects between the visual stimulus and the instructions.

The temporal stimulus was a sinusoidally modulated light-emitting diode (LED; Hewlett Packard, HLMP-3750) that was blurred with a low-pass spatial filter. The LED was placed behind an optical display with the spatial frequency filtering properties of parallel diffusive plates. The diffuser plates, made of frosted glass $0.32 \mathrm{~cm}$ thick, were $2.54 \mathrm{~cm}$ apart. This distance, multiplied by the spatial frequencies of the LED at the rear diffuser plate, determined the spatial frequencies of the LED visible to the observer. ${ }^{1}$ The idea of a pure temporal stimulus is an experimental construct; in reality, any presentation of a temporal stimulus will have a spatial component. In this experiment, a low-pass spatial filter was used, since it is known that both visually effective edges and high spatial frequencies in the target elevate the sensitivity to low temporal frequencies (Watson, 1986).

In Phase 1, the temporal signals, sinusoidal variations in luminance, were controlled by a wave generator (Wavetek Programmable Generator, Model 154). In the other three phases, the signals were generated by an Apple Ile microcomputer. The sinusoidal variations in luminance began at a zero-crossing and were embedded in a 2-sec Gaussian envelope. ${ }^{2}$ A photocell unit monitored the LED's light output; its mean luminance was $11.7 \mathrm{fL}$, and its peakto-trough modulation was $11.24 \mathrm{fL}\left(L_{\max } 17.32 \mathrm{fL}\right.$ and $L_{\min }$ $6.08 \mathrm{fL}$ ). The reference contrast of the $\mathrm{LED}$, given by Michelson contrast $\left[\left(L_{\max }-L_{\min }\right) /\left(L_{\max }+L_{\min }\right)\right]$, was 0.48 . In this experiment, contrast is also represented in terms of the amount of attenuation a particular pattern undergoes with respect to this reference contrast; all signals were attenuated on a logarithmic scale (in decibels) with reference to this value.

The circular LED display subtended $1^{\circ}$ visual angle and appeared superimposed on a wide ( $15^{\circ}$ visual angle in diameter) circular homogeneous field illuminated by wide band ("white") light. The luminance of this field was $16 \mathrm{fL}$, measured by a photometer (Soligor Digital Spot Sensor Meter, Model 401). An LED was used for two reasons: It has a fast response and thus follows the applied waveform closely, and it emits narrow band light whose relative spectral composition is independent of intensity (Moulden, Renshaw, \& Mather, 1984). The LED had a primary wavelength of $635 \mathrm{~nm}$ at peak, which essentially affects only red receptors. Had a wide spectrum light source been used, the activation of an observer's color pathways of different temporal responsiveness could have confounded the results (Nilsson, Richmond, \& Nelson, 1975).

In Phase 1, observers used an attenuator set equipped with 1-dB steps (Hewlett Packard 350 D), to adjust the amplitude modulation of the sinusoidal variation in luminance of the LED. In the other phases, the observers indicated whether or not they had detected a signal by using pushbuttons connected to the microcomputer. Throughout the experiment, the investigator monitored the stimulus presentation and the observers' adjustment settings with an oscilloscope (Tektronix $502 \mathrm{~A}$ dual-beam).

The observers sat in a dark room, $200 \mathrm{~cm}$ away from a screen onto which slides with the spatial adaptation fields were rearprojected. These patterns consisted of vertical sinusoidal gratings of high (10.3-cpd) or low (0.6-cpd) spatial frequencies. ${ }^{3}$ The gratings had a wide spectral composition in which the region corresponding to the primary wavelength of the LED was well represented. The slides are of low harmonic distortion at approximately 0.6 contrast. The light bars of the gratings had a luminance, $L_{\max }$, of $12 \mathrm{fL}$, and the dark bars a luminance, $L_{\min }$, of $3 \mathrm{fL}$.

The experiment took place in a dark room. At the beginning of every experimental session, the observers first adapted to darkness for $5 \mathrm{~min}$. They then viewed the temporal stimulus monocularly through an artificial pupil with an aperture diameter of $1.8 \mathrm{~mm}$, 
while the other eye was occluded by an eyepatch. The observers' head movements were restricted by a chinrest.

\section{Phase 1}

Observers adjusted the modulation amplitude of the LED to define their threshold for temporal frequencies ranging from 0.5 to $12 \mathrm{~Hz}$. The purpose of this phase was to see whether the LED employed here would yield TCSFs similar to those obtained by other investigators who used somewhat different displays. The experiment would also indicate whether the observers had normal sensitivity to temporal patterns.

\section{Method}

Observers. Four Princeton University students with normal or corrected-to-normal vision were paid for their participation in all four phases. They had not participated in related experiments and were naive as to the purpose of the experiment. This phase was conducted in a 1-h session on 2 consecutive days.

Procedure. To obtain TCSFs, the observers were asked to look at the LED as its intensity varied sinusoidally at a particular frequency. They were instructed to adjust the amplitude in 1-dB steps until the intensity modulation looked "just barely detectable' (just before the light seemed steady in brightness).

The LED was sinusoidally modulated at 11 different temporal frequencies: $0.5,1,1.5,2,4,6,8,10,11,11.5$, and $12 \mathrm{~Hz}$. On each of 2 successive days, the observers adjusted the amplitude of each frequency at flicker threshold five times. The 11 frequencies were randomly ordered in each of 10 blocks. At the beginning of both sessions, the observers performed a practice block.

\section{Results}

Figure 1 presents the averaged TCSF for each observer and one averaged across the 4 observers. This figure shows that these results are similar to those obtained by
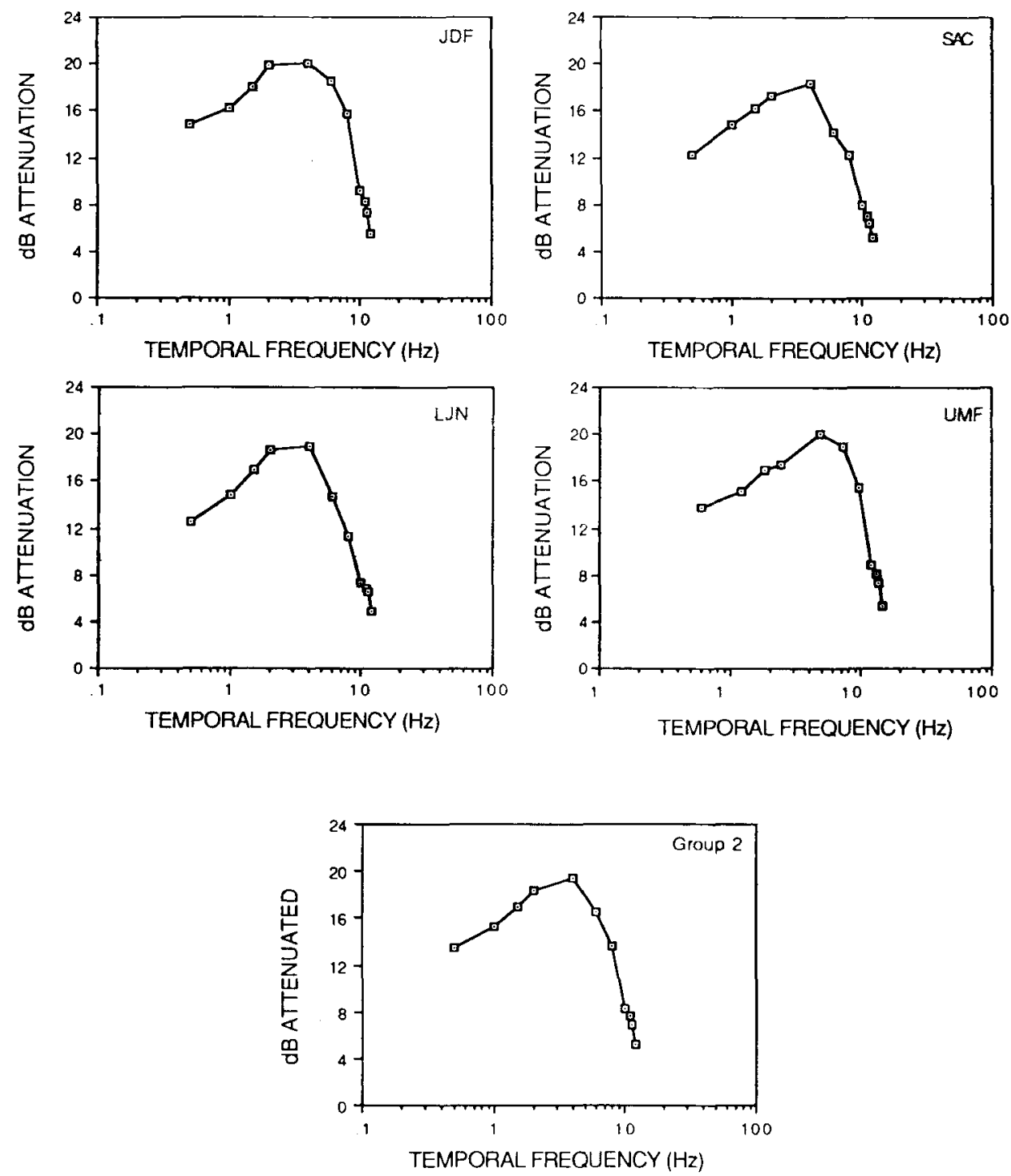

Figure 1. Temporal contrast sensitivity functions for each observer, and averaged across the 4 observers. 
other investigators (e.g., Kelly, 1961); that is, more contrast was required to perceive "flickering" at the lower and higher frequencies of the curve and less contrast at the middle frequencies.

\section{Phase 2}

The purpose of this phase was to identify the appropriate amplitudes of both a 2- and a $12-\mathrm{Hz}$ temporal pattern that would be just discriminable from a steady light source $(0 \mathrm{~Hz})$ at $d^{\prime}$ values $\approx 1.5$. Phase 2 took place in eight 1-h sessions.

\section{Method}

At the beginning of all sessions, the observers were instructed to perform a practice block of 50 trials of the yes/no detection task. The beginning of each trial was indicated to the observers by the sounding of a 2 -sec tone. During this sample interval, the LED display was always modulated to define a signal, a variation in LED luminance. This both alerted the observers to the onset of the trial and indicated exactly what a signal looked like. One second later, the tone again sounded for $2 \mathrm{sec}$ to define the test interval during which either a signal was again presented or not (in a random $50 \%$ of the trials). Two seconds after the yes/no response the computer initiated the next trial. The observers' responses were self-paced; they typically responded within $1 \mathrm{sec}$. They were told that the signal would occur in a random $50 \%$ of the trials.

At each of the eight sessions, the observers performed three blocks of 100 trials, consisting of four sessions of $12-\mathrm{Hz}$ signals and four sessions of $2-\mathrm{Hz}$ signals in a counterbalanced order.

\section{Results}

At the end of the eight sessions, the percentages of the waveform amplitude needed to yield the $d^{\prime} \approx 1.5$ value for the 2- and 12-Hz signals were as follows, respectively: $5.8 \%$ and $23 \%$ for observer J.D.F., $12 \%$ and $45 \%$ for S.C.A., $10 \%$ and $51 \%$ for L.J.N., and $6.5 \%$ and $25 \%$ for U.M.F.

\section{Phase 3}

In this phase, the effects of adaptation to spatial sinusoidal gratings on sensitivity to temporal patterns were investigated, using the yes/no detection task. The observers were adapted to either a high- or a low-spatial-frequency pattern, and their sensitivity to high or low temporal frequencies was measured and compared with their sensitivity before adaptation. This phase was conducted in 12 1-h sessions.

\section{Method}

Stimuli and Apparatus. The amplitudes of the high and low temporal frequencies ( 12 and $2 \mathrm{~Hz}$ ) yielded a comparable $d^{\prime} \approx 1.5$ for each observer before adaptation. The spatial patterns were vertical sinusoidal gratings of high $(10.3 \mathrm{cpd})$ or low $(0.64 \mathrm{cpd})$ frequencies, rear-projected onto a screen.

Procedure. A within-subjects factorial design was utilized. All observers participated in four types of experimental conditions, one per session. They adapted to a high- or a low-spatial-frequency grating, and were subsequently tested at both temporal frequencies, high and low. Following the preadaptation block, the observers were instructed to scan the adaptation grating slowly and continuously back and forth for the duration of its presentation. A postadaptation block lasted approximately $4 \mathrm{~min}$, immediately following the 4-min adaptation period. The detection task was identical to that described in Phase 2. There were also two control conditions, in which observers adapted to a homogeneous field ( $0 \mathrm{cpd}$ ) with the same mean luminance as the adaptation grating and were subsequently tested at each temporal frequency.

Each observer yielded five blocks of 50 trials per session: two pre- and three postadaptation. There were 2 sessions per condition. The order of presentation of the 12 sessions ( 2 sessions of 2 temporal frequencies per 3 spatial adaptation fields) was counterbalanced among observers. At the beginning of all sessions, the observers performed a practice block of 50 trials.

\section{Results}

For each observer, the average was computed for the $d^{\prime}$ values obtained in the two pre- and the three postadaptation blocks for each of the 12 sessions. The $d^{\prime}$ 's of the two sessions for the same condition were also averaged. The adaptation effect was assessed by comparing the $d^{\prime}$ 's for the two low and two high temporal frequencies, in the pre- and postadaptation conditions, for gratings of high and low spatial frequencies (see Figure 2).

The $d^{\prime}$ 's of the four experimental conditions were subjected to a within-subjects, three-way ANOVA: adapting spatial frequency (low vs. high) $\times$ testing temporal frequency (low vs. high) $\times$ adaptation (pre- vs. postadaptation). The overall interaction between adaptation and temporal frequency was significant $[F(1,3)=14.89$, $p<.05$ ], as were the interactions for both the high- and the low-spatial-frequency gratings $[F(1,3)=110.51$, $p<.001$, and $F(1,3)=5.82, p<.10$, respectively]. Specifically, there was a significant difference between pre- and postadaptation sensitivities to the $2-\mathrm{Hz}$ signal when the adaptation stimulus was a low-frequency grating $[F(1,3)=42.61, p<.01]$, but not when it was a high-frequency grating $[F(1,3)=0.29]$. Conversely, there was a significant difference between pre- and postadaptation sensitivities to the $12-\mathrm{Hz}$ signal when the adaptation stimulus was a high-frequency grating $[F(1,3)=$ $13.40, p<.05$ ], but not when it was a low-frequency grating $[F(1,3)=0.27]$.

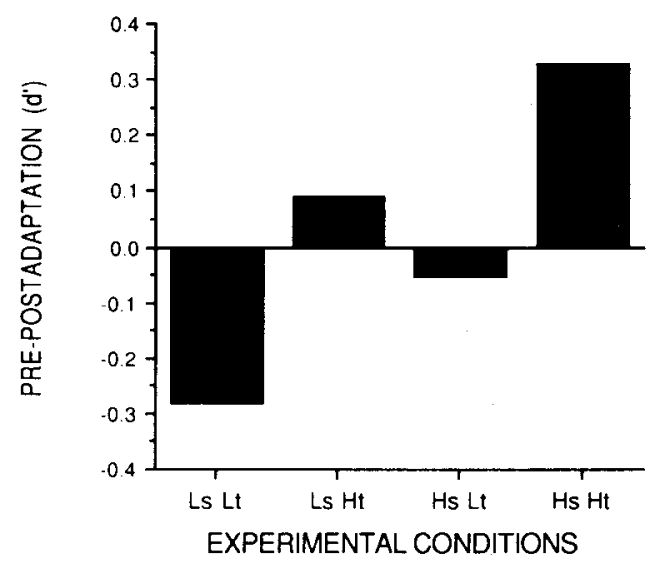

Figure 2. Change of the mean d' values before and after adaptation to a low-spatial-frequency grating ( $L s ; 0.6 \mathrm{cpd}$ ) or a high-spatialfrequency grating (Hs; 10.3 cpd), for a low temporal frequency (Lt; $2 \mathrm{~Hz}$ ) and a high temporal frequency (Ht; $12 \mathrm{~Hz}$ ). 
A $t$ test for correlated groups was performed to compare pre- versus postadaptation sensitivities $\left(d^{\prime}\right)$ in the control condition (i.e., adaptation to a homogeneous field). The difference in $d$ 's was significant for neither the lowtemporal-frequency stimulus $[t(3)=0.81]$, nor the hightemporal-frequency stimulus $[t(3)=-0.07]$. These results show that it was the properties of the gratings, and not merely luminance level, that produced the changes in sensitivity reported above.

SDT has provided a theoretical framework for the analyses conducted so far. The data obtained in this phase, however, were also analyzed in a nontheoretical way: the probabilities of correct responses (\%CR) of the four experimental conditions were subjected to a three-way ANOVA. The results were the same as those obtained with $d^{\prime}$.

To summarize, the analyses presented here, \%CR and $d^{\prime}$, as well as preliminary data obtained with the method of adjustment, all had exactly the same implications. Adaptation to the low-spatial-frequency grating significantly decreased sensitivity to the low-temporal-frequency stimulus; however, it did not affect the observers' sensitivities to the high-temporal-frequency stimulus. Moreover, a surprising result was also found: Adaptation to the high-spatial-frequency grating significantly increased sensitivity to the high-temporal-frequency stimulus; it did not affect sensitivity to the low-temporal-frequency stimulus. None of the control conditions yielded significant adaptation effects.

\section{Phase 4}

The purpose of Phase 4 was to determine whether there were additive effects between the visual stimulus and the instructions. An ROC was generated by asking observers to perform the yes/no detection task under instructional conditions designed to induce a "liberal," " neutral," or "conservative" judgmental standard for reporting signals. If the results obtained here would be better described by the "unequal variance model" than by the "equal variance model" of SDT (see the Discussion section), they would then be the basis of a theoretical "correction factor" to reanalyze the results of Phase 3.

\section{Method}

Stimuli and Apparatus. Two temporal frequencies were used, 2 and $12 \mathrm{~Hz}$, at the amplitudes obtained in Phase 2-that is, where they yielded similar discriminability values for each observer of $d^{\prime} \approx 1.5$. The experiment setting was the same as in Phase 2 .

Procedure. A $3 \times 2$ within-subjects factorial design was utilized; all observers participated in three instructional conditions-liberal, neutral, or conservative-and were tested at both temporal frequencies, high $(12 \mathrm{~Hz})$ and low $(2 \mathrm{~Hz})$, in the yes/no detection task (see Phase 2).

The instructional conditions consisted of informing the observer how to regard the relative importance of hits $[\mathrm{R} 1 \mid \mathrm{S} 1]$ and false alarms [FA; R1|S0]-that is, the typical instructions to manipulate the criterion to report signals. To induce a liberal or a conservative judgmental standard, the observers were asked to report a signal (S1) when they either had a slight indication or were very certain that the LED had flickered. To induce a neutral judgmental standard, the observers were told to maintain the same criterion they had in Phases 2 and 3, when they kept in mind that the LED would flicker in $50 \%$ of the trials.

All observers participated in four 1-h sessions, two with the high and two with the low temporal frequency, 12 and $2 \mathrm{~Hz}$, respectively. Each session consisted of a practice block and six blocks of $\mathbf{5 0}$ trials; two blocks under each of three instructions. This yielded 600 trials on which to base estimates of the probability of a "yes" response for each temporal frequency. The presentation order of the instructional conditions was counterbalanced among observers and experimental sessions.

\section{Results}

For each observer, the average was obtained of the \%hit and \%FA yielded under the three instructional conditions-liberal, neutral, or conservative-for both temporal frequencies, high and low. The results of the two sessions for the same condition were also averaged for each observer. The \%hit and \%FA for 2 and $12 \mathrm{~Hz}$ were converted into $z$ scores (Figure 3 ).

For each temporal frequency, the $z$ scores of the six experimental conditions were subjected to a within-

a) $2 \mathrm{~Hz}$

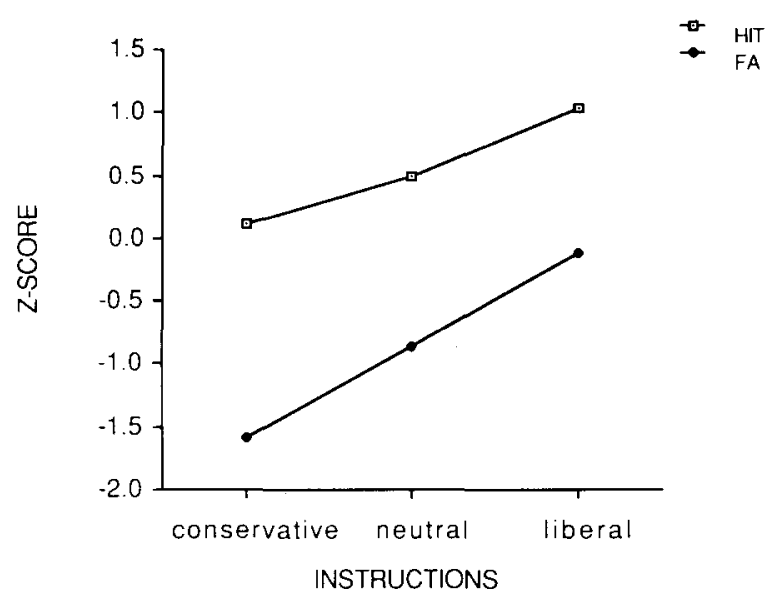

b) $12 \mathrm{~Hz}$

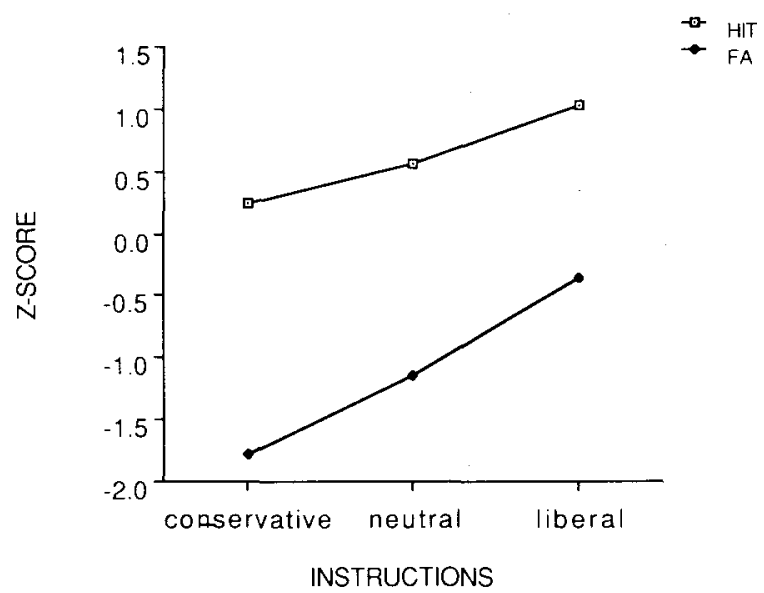

Figure 3. Group $z$ scores under conservative, neutral, and liberal instructions (a) for a low temporal frequency $(2 \mathrm{~Hz}$ ) and (b) for a high temporal frequency $(12 \mathrm{~Hz})$. 
subjects, two-way ANOVA: instructional condition $\times$ stimulus (\%hit vs. \%FA). For both temporal frequencies, stimulus and instructions had a significant effect on performance [Stimulus: $F(1,3)=82.10, p<.005$, for the lowand $F(1,3)=539.22, p<.001$, for the high-temporalfrequency stimulus. Instructions: $F(2,6)=36.78, p<$ .001 , for the low- and $F(2,6)=126.09, p<.001$, for the high-temporal-frequency stimulus].

Instructions and stimulus interacted significantly at both temporal frequencies $[F(2,6)=9.33, p<.05$, for the low and $F(2,6)=16.24, p<.005$, for the high temporal frequency], and both factors were significant at all levels: Instructions had a significant effect on hits and FA at both frequencies [on hits: $F(2,6)=18.32, p<.005$, at the low and $F(2,6)=76.37, p<.001$, at the high temporal frequency. On FA: $F(2,6)=45.28, p<.001$, at the low and $F(2,6)=90.78, p<.001$, at the high temporal frequency]. Likewise, stimulus had a significant effect on the three instructions: liberal $[F(1,3)=201.28, p<.001$, for the low and $F(1,3)=235.8, p<.001$, for the high temporal frequency]; neutral $[F(1,3)=37.76, p<.01$, for the low and $F(1,3)=165.41, p<.001$, for the high temporal frequency]; and conservative $[F(1,3)=88.25$, $p<.005$, for the low and $F(1,3)=445.27, p<.001$, for the high temporal frequency].

According to the equal variance model of SDT, a $z$ transformation of \%hits and \%FA should reveal an additive effect of stimulus and instructions. Given that a significant interaction was found-there was no additivity effect-the results obtained in this phase of the experiment were the basis of a theoretical correction factor used to conduct a post hoc analysis of the results obtained in Phase 3.

\section{Reanalysis of Phase 3 on the Basis of Phase 4}

It is not totally clear what factors cause certain detection tasks to yield ROC functions with slopes less than one; it is worth noting, however, that visual signals generally tend to yield such functions (D. M. Green \& Swets, 1966). Such data suggest that the variance of $X$ ("'noise") on signal trials is greater than on nonsignal trials; that is, $\operatorname{Var}(\mathrm{X} \mid \mathrm{S} 1)>\operatorname{Var}(\mathrm{X} \mid \mathrm{S} 0)$.

The data from Phase 4 show that the four observers had ROC functions with slopes of less than one $(k<1)$, indicating that the unequal variance model of SDT would be required to adequately characterize the observers' performances. The earlier analysis of data from Phase 3 might have spuriously shown shifts in sensitivity $\left(d^{\prime}\right)$ that would more appropriately be interpreted as shifts in criterion along an unequal variance $(k<1)$ ROC function due to judgmental factors.

Consequently, it was necessary to reanalyze the data from Phase 3 by correcting the differences in pre- and postadaptation performance using the new values of $k$ less than one $(k<1)$. These values (Figure 4), obtained by means of the least squared error solution, were used to calculate new estimates of $d^{\prime}$ in the pre- and postadaptation conditions of Phase 3. These new estimates, designated as $d^{\prime \prime}$, were subjected to a within-subjects, three-way ANOVA: adapting spatial frequency $(0.6 \mathrm{vs} .10 .3 \mathrm{cpd})$ $\times$ temporal frequency of target $(2 \mathrm{vs} .12 \mathrm{~Hz}) \times$ adaptation (pre- vs. postadaptation).

The mean differences between pre- and postadaptation estimates of temporal sensitivity in the various experimental conditions are shown graphically in Figure 5. There was a significant difference between pre- and postadaptation to the low-spatial-frequency grating for the lowtemporal-frequency stimulus $[F(1,3)=22.14, p<.01]$, but not for the high-temporal-frequency stimulus $[F(1,3)=$ $0.35]$. Conversely, there was a significant difference between pre- and postadaptation to the high-spatial-frequency grating for the high-temporal-frequency stimulus $[F(1,3)=$ $12.10, p<.05$ ], but not for the low-temporal-frequency stimulus $[F(1,3)=0.22]$. Adaptation interacted significantly with temporal frequency $[F(1,3)=14.21, p<$ $.05]$. This interaction indicated that the adaptation effect was significant after adaptation to the high-spatialfrequency grating $[F(1,3)=90.38, p<.001]$, and marginally significant after adaptation to the low-spatialfrequency grating $[F(1,3)=5.75, p<.10]$.

In summary, then, the basic pattern of results obtained with the equal variance model in Phase 3 , as well as with a reanalysis of these data based on the ROC functions defined in Phase 4 (unequal variance model), remains the same: sensitivity to low-frequency temporal signals was lowered by adaptation to low-spatial-frequency gratings, and unaffected by adaptation to high-spatial-frequency gratings; whereas, sensitivity to high-frequency temporal signals was increased by adaptation to high-spatialfrequency gratings, and unaffected by adaptation to lowspatial-frequency gratings.

\section{GENERAL DISCUSSION}

SDT provides an analytic technique for measuring an observer's sensory capacities and judgmental criteria separately. Most applications of SDT assume that there are additive effects of the visual stimulus and instructions; the variance in the signal (S1) and no-signal (SO) distributions are the same, or in other words, the ROC function has a slope of $1(k=1)$. This form of SDT, called the equal variance model, since $\operatorname{Var}(\mathrm{X} \mid \mathrm{S} 1)$ equals $\operatorname{Var}(\mathrm{X} \mid \mathrm{S} 0)$, yields a measure of sensitivity $d^{\prime}$ whose value is uninfluenced by variations in judgmental standards.

If, however, there is a slight interaction between the visual stimulus and the judgmental standards (i.e., if these effects are not additive), the unequal variance model would describe the data in a more appropriate fashion. This slightly more complex form of the model differs only in that $\operatorname{Var}(X \mid S 1)$ need not equal $\operatorname{Var}(X \mid S 0)$; hence, it requires two parameters to characterize sensory capacity, $d^{\prime}$ and a parameter $k$ indicating the relation between $\operatorname{Var}(\mathrm{X} \mid \mathrm{S} 1)$ and $\operatorname{Var}(\mathrm{X} \mid \mathrm{S} 0)$. Even though it has been shown that visual signals generally yield ROC functions with slopes of less than $1(k<1$; D. M. Green \& Swets, 1966), many researchers have not tested for additivity. 


\section{a) $2 \mathrm{~Hz}$}
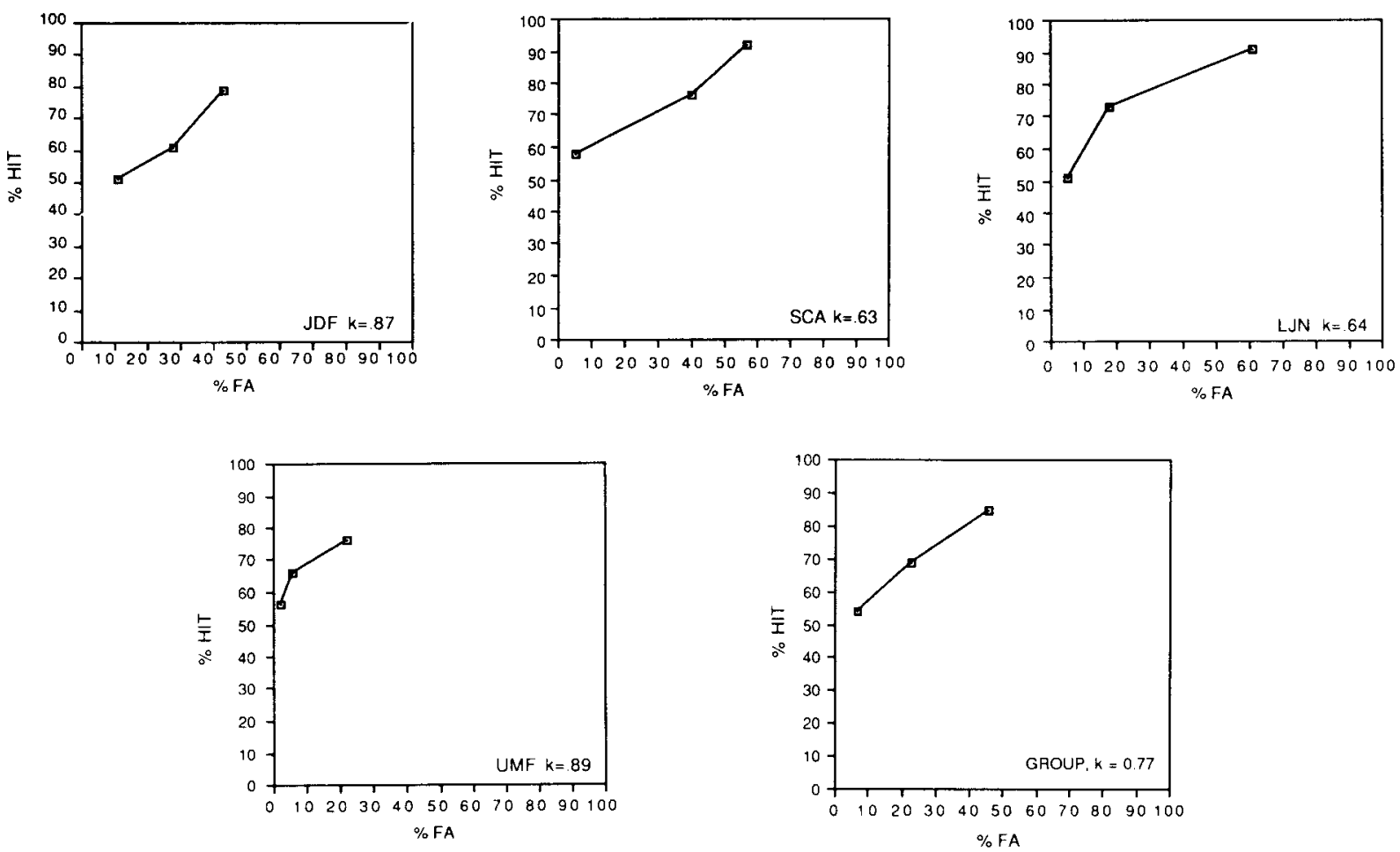

\section{b) $12 \mathrm{~Hz}$}
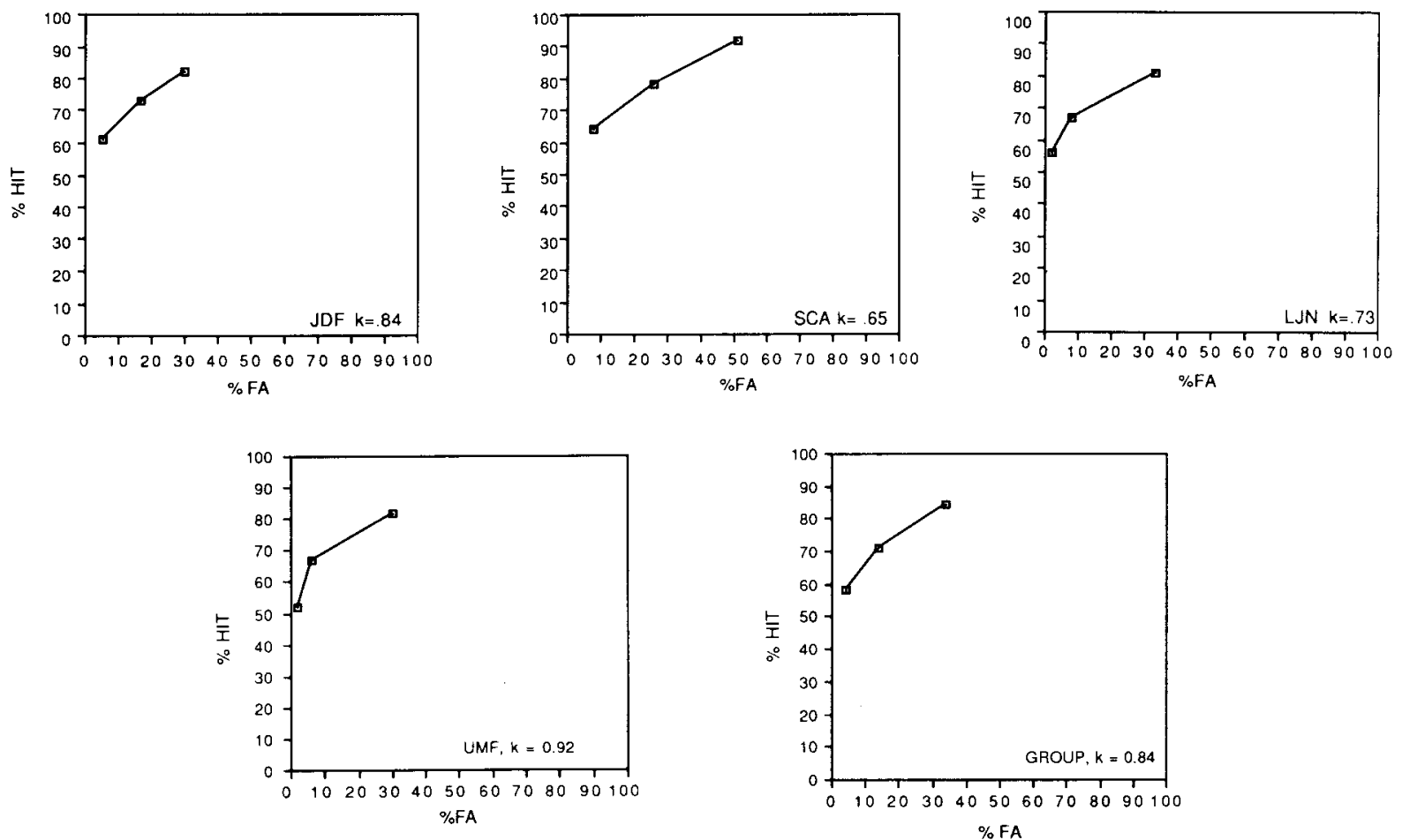

Figure 4. Unequal variance receiver operating characteristic functions for each observer and averaged across the 4 observers for (a) a low temporal frequency $(2 \mathrm{~Hz})$, and (b) a high temporal frequency $(12 \mathrm{~Hz})$. Note that slope $k<1$. 


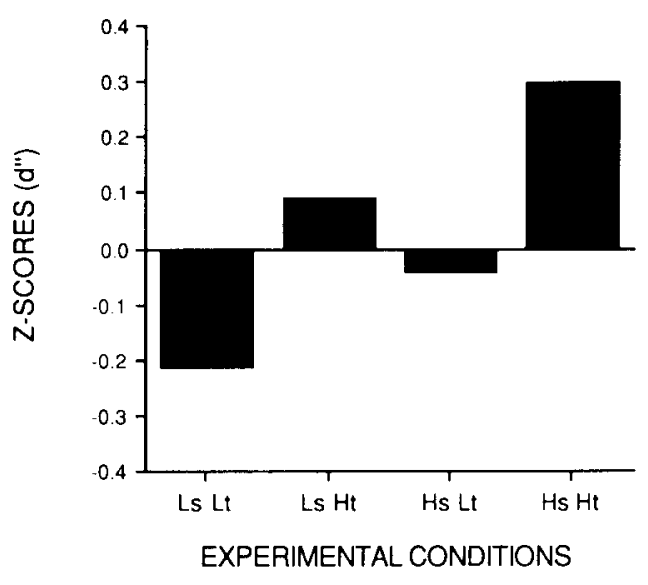

Figure 5. Change of the mean $d^{\prime \prime}$ (corrected $d^{\prime}$ ) values before and after adaptation to a low-spatial-frequency grating (Ls; 0.6 cpd) or a high-spatial-frequency grating (Hs; 10.3 cpd), for a low temporal frequency $(\mathrm{Lt} ; 2 \mathrm{~Hz}$ ) and a high temporal frequency $(\mathrm{Ht} ; 12 \mathrm{~Hz})$.

In the present study, the results of Phase 4 showed that the unequal variance model explained the data of Phase 3 more accurately than the equal variance model; on the basis of this finding, a reanalysis of the results was carried out. The same major effects were found. Furthermore, the results obtained with the yes/no detection task of SDT replicated those obtained in a preliminary study in which the psychophysical method of adjustment was employed.

In general, an interaction is said to occur when the perception of the combination of two or more stimuli or stimulus components differs from what would be expected if each component were perceived independently of the other. The presence of an interaction, signaled by a change in threshold, is taken as evidence that the two stimuli share at least some processing pathways. Conversely, the absence of an interaction is taken as evidence that the stimuli are processed by separate, and in some cases independent, neural pathways (see, e.g., Olzak \& Thomas, 1986). In the present study, when observers adapted to a lowspatial-frequency grating, they experienced a decrease in sensitivity to low temporal frequencies (i.e., an inhibitory effect was found), whereas when observers adapted to a high-spatial-frequency grating, they experienced an increase in sensitivity to high temporal frequencies (i.e., a facilitatory effect was found).

The inhibitory effect of the low spatial on low temporal frequencies was expected. The selective adaptation effect is often defined as a reduction in sensitivity to subsequent stimulation of the same sort after prolonged exposure to a particular stimulus. This result supports the inseparability at low frequencies. In contrast, the facilitatory effect of the high spatial on high temporal frequencies, which suggests inseparability at high frequencies, was surprising. There are, however, some instances of adaptation to one stimulus that result in an increase in sensitiv- ity to other stimuli; lateral interactions among retinal elements are an example. This adaptation effect "disinhibits" a preadaptation inhibition; the net effect is a heightened sensitivity. One possible interpretation of the facilitatory effect of adaptation to high-spatial-frequency targets on sensitivity to high-temporal-frequency modulation is that of mutually inhibitory systems. Specifically, activity in the high spatially tuned system might inhibit the activity of a high temporally tuned system. Adapting the high spatially tuned system could weaken its response and thereby reduce its inhibitory effect on the high temporal system.

Although the explanation above is post hoc, there is evidence for such facilitatory interactions. Contrast sensitivity to frequencies up to two or three octaves away from the adaptation frequency may actually be increased by spatial adaptation (K. K. DeValois, 1977). This result suggests that spatial frequency channels are not truly independent, but rather that they may be mutually inhibitory. The assumption of independence has also been challenged by the results of some studies in masking (Tolhurst \& Barfield, 1978) and in summation (Hirsch, Hylton, \& Graham, 1982; Olzak, 1986).

Even though the inhibitory interactions revealed by such studies are relatively minor when compared to the large loss of sensitivity at and around the adaptation frequency, they nonetheless allow us to understand better the physiological and psychophysical interactions and the organization underlying spatial-frequency-specific channels (R. L. DeValois \& K. K. DeValois, 1988). Whereas the inhibitory interactions found in these studies have been reported within the spatial domain, they have not, to my knowledge, been reported either in the temporal domain or between the spatial and the temporal domains.

The evidence on the separability/interaction issue is equivocal and puzzling; nevertheless, some authors assume that the spatial and temporal sensitivities are separable and proceed to report sensitivity measurements or to propose models of spatial vision (e.g., Wilson \& Gelb, 1984) and motion perception (e.g., Harris, 1986) based on that assumption. Given the results of the present study and others supporting inseparability (e.g., Fleet et al., 1985), it seems that: (1) models based on the assumption of separability should be reviewed; (2) measurements of either spatial or temporal sensitivities would have to take the other domain into account; and (3) care must be taken in generalizing from experiments in spatial vision when only a single value of temporal or spatial stimulation has been used-or, at the very least, information about the other factor ought to be reported.

\section{REFERENCES}

Blakemore, C., C Campbell, F. W. (1969). On the existence of neurons in the human visual system selectively sensitive to the orientation and size of retinal images. Vision Research, 203, 237-260.

Carlson, C. R., Heyman, P. M. (1979). A large format optical display for the generation of generalized psychophysical stimuli. Vision Research, 19, 99-103. 
Dawis, S., Shapley, R., Kaplan, E., \& Tranchina, D. (1984). The receptive field organization of $\mathrm{X}$ cell in the cat: Spatiotemporal coupling and asymmetry. Vision Research, 24, 549-564.

Derrington, A. M., LenNie, P. (1982). The influence of temporal frequency and adaptation level on the receptive field organization of retinal ganglion cells in the cat. Joumal of Physiology, 333, 343-366.

DEVALOIS, K. K. (1977). Spatial frequency adaptation can enhance contrast sensitivity. Vision Research, 17, 1057-1066.

DeValois, R. L., \& DeVAlois, K. K. (1988). Spatial vision. New York: Oxford University Press.

Enroth-Cugell, C., LenNie, P. (1975). The control of retinal ganglion cell discharge by receptive field surround. Journal of Physiology, 247, 551-578.

FleEt, D. T., Hallett, P. E., \& JEPSON, A. D. (1985). Spatiotemporal inseparability in early visual processing. Biological Cybernetics, 52 , 153-164.

Graham, N. (1985). Detection and identification of near-threshold visual patterns. Journal of the Optical Society of America A, 2, 1468-1482.

Graham, N., Robson, J. G., \& Nachmias, J. (1978). Grating summation in fovea and periphery. Vision Research, 18, 815-826.

Green, D. M., \& SweTS, J. A. (1966). Signal detection theory and psychophysics. New York: Wiley.

GREEN, M. (1981). Psychophysical relationships among mechanisms sensitive to pattern, motion and flicker. Vision Research, 21, 971-983.

HARRIS, M. G. (1984). The role of pattern and flicker mechanisms in determining the spatiotemporal limits of velocity perception of upper movement thresholds. Perception, 13, 401-407.

HaRRIS, M. G. (1986). The perception of moving stimuli: A model of spatiotemporal coding in human vision. Vision Research, 26, 1281-1287

Hess, R., \& Plant, G. T. (1985). Temporal frequency discrimination in human vision: Evidence for an additional mechanism in the low spatial and high temporal frequency region. Vision Research, 25, 1493-1500.

Hirsch, J., Hylton, R., \& Graham, N. (1982). Simultaneous recognition of two spatial frequency components. Vision Research, 22, 365-375.

KELLY, D. H. (1961). Visual responses to time-dependent stimuli: I. Amplitude sensitivity measurements. Journal of the Optical Society of America, 51, 422-429.

KELLY, D. H. (1966). Frequency doubling in visual responses. Journal of the Optical Society of America, 56, 1141.

KoEnderink, J. J., \& VAN DOORN, A. J. (1979). Spatiotemporal contrast detection threshold surface is bimodal. Optics Letters, 4, 32-34.

LEHKY, S. R. (1985). Temporal properties of visual channels measured by masking. Journal of the Optical Society of America, 2, 1260-1272.

Moulden, B., Renshaw, J., \& Mather, G. (1984). Two channels for flicker in the human visual system. Perception, 13, 387-400.

Nilsson, T. H., Richmond, C. F., \& Nelson, T. M. (1975). Flicker adaptation shows evidence of many visual channels selectively sensitive to temporal frequency. Vision Research, 15, 621-624.

OlzaK, L. A. (1986). Widely separated spatial frequencies: Mechanism interactions. Vision Research, 26, 1143-1153.

OlzaK, L. A., \& Thomas, J. P. (1986). Seeing spatial patterns. In K. R. Boff, L. Kaufman, \& J. Thomas (Eds.), Handbook of perception and performance (Vol. 1, chap. 7, pp. 1-56). New York: Wiley.

PANTle, A. (1970). Adaptation to pattern spatial frequency: Effects on visual movement sensitivity in humans. Journal of the Optical Society of America, 60, 1120-1124.

PARKER, A. (1981). Shifts in perceived periodicity induced by temporal modulation and their influence on the spatial frequency tuning of two aftereffects. Vision Research, 21, 1739-1747.

RoBSon, J. G. (1966). Spatial and temporal contrast sensitivity functions of the visual system. Journal of the Optical Society of America, 56, 1141-1142.

SmITH, R. A. (1970). Adaptation of visual contrast sensitivity to specific temporal frequencies. Vision Research, 10, 275-279.

Tolhurst, D. J., \& BARField, L. P. (1978). Interactions between spatial frequency channels. Vision Research, 18, 951-958.

Tolnurst, D. J., \& Movshon, J. A. (1975). Spatial and temporal contrast sensitivity of striate cortical neurons. Nature, 257, 674-675.

Watson, A. B. (1986). Temporal sensitivity. In K. R. Boff, L. Kaufman, \& J. Thomas (Eds.), Handbook of perception and performance (Vol. 1, chap. 6, pp. 1-43). New York: Wiley.

WILSON, H. R. (1980). Spatiotemporal characterization of a transient mechanism in the human visual system. Vision Research, 20, 443-452.

Wilson, H. R., \& GeLB, D. J. (1984). Modified line element theory for spatial-frequency and width discrimination. Journal of the Optical Society of America A, 1, 124-131.

\section{NOTES}

1. The bandpass characteristics of the device are characterized by $x f$, where $x$ is the plate spacing and $f$ is the spatial frequency at the rear diffuser plate. The modulation transfer function, $R$, is represented by the analytical expression: $R=\left[1.0+1.43(x f)^{2}+0.168(x f)^{6}\right]^{-1}$ (Carlson \& Heyman, 1979).

2 . According to the formula describing the luminance profile of a sinewave grating truncated by multiplication with a Gaussian function (Graham, Robson, \& Nachmias, 1978).

3. A stationary grating is not a purely spatial stimulus. Stabilized retinal image experiments have shown that spatial discrimination requires temporal illumination changes on the retina. Under unstabilized conditions, these changes are generated by spatial translations of the retinal image by eye movements, or in this experiment by scanning.

(Manuscript received January 22, 1990; revision accepted for publication July 9, 1990.) 